\title{
Functional Magnetic Resonance Imaging-Assessed Brain Responses during an Executive Task Depend on Interaction of Sleep Homeostasis, Circadian Phase, and PER3 Genotype
}

\author{
Gilles Vandewalle, ${ }^{1}$ Simon N. Archer, ${ }^{2}$ Catherine Wuillaume, ${ }^{1}$ Evelyne Balteau, ${ }^{1}$ Christian Degueldre, ${ }^{1}$ André Luxen, ${ }^{1}$ \\ Pierre Maquet, ${ }^{1}$ and Derk-Jan Dijk ${ }^{2}$ \\ ${ }^{1}$ Cyclotron Research Centre, University of Liège, B-4000 Liège, Belgium, and ${ }^{2}$ Surrey Sleep Research Centre, University of Surrey, Guildford, Surrey GU2 \\ 7XP, United Kingdom
}

\begin{abstract}
Cognition is regulated across the $24 \mathrm{~h}$ sleep-wake cycle by circadian rhythmicity and sleep homeostasis through unknown brain mechanisms. We investigated these mechanisms in a functional magnetic resonance imaging study of executive function using a working memory 3-back task during a normal sleep-wake cycle and during sleep loss. The study population was stratified according to homozygosity for a variable-number ( 4 or 5 ) tandem-repeat polymorphism in the coding region of the clock gene PERIOD3. This polymorphism confers vulnerability to sleep loss and circadian misalignment through its effects on sleep homeostasis. In the less-vulnerable genotype, no changes were observed in brain responses during the normal-sleep wake cycle. During sleep loss, these individuals recruited supplemental anterior frontal, temporal and subcortical regions, while executive function was maintained. In contrast, in the vulnerable genotype, activation in a posterior prefrontal area was already reduced when comparing the evening to the morning during a normal sleep-wake cycle. Furthermore, in the morning after a night of sleep loss, widespread reductions in activation in prefrontal, temporal, parietal and occipital areas were observed in this genotype. These differences occurred in the absence of genotype-dependent differences in circadian phase. The data show that dynamic changes in brain responses to an executive task evolve across the sleep-wake and circadian cycles in a regionally specific manner that is determined by a polymorphism which affects sleep homeostasis. The findings support a model of individual differences in executive control, in which the allocation of prefrontal resources is constrained by sleep pressure and circadian phase.
\end{abstract}

\section{Introduction}

The human brain can uphold cognitive activity throughout a waking day via a putative arousal signal driven by the circadian pacemaker (Czeisler and Dijk, 2001; Dijk and von Schantz, 2005), which counters the progressive increase in homeostatic sleep pressure (Cajochen et al., 2002). When wakefulness is extended into the circadian night, cognitive function is jeopardized, because the circadian arousal signal dissipates (Dijk et al., 1992). The vulnerability to the effect of sleep loss differs markedly between individuals and does so in a trait-like manner (Van Dongen et al., 2004). The brain bases for maintenance of cognition during the day, its deterioration during the night and individual

Received Jan. 14, 2009; revised April 15, 2009; accepted April 24, 2009.

This work was supported by the Belgian Fonds de la Recherche Scientifique (FNRS), the Fondation Médicale Reine Elisabeth, the University of Liège, the Pôles d'Attraction Interuniversitaire/Interuniversity Attraction Poles (Grant P6/29), and by the Wellcome Trust (Grant GR069714MA) and the Biotechnology and Biological Sciences Research Council (Grant BB/F022883/1) (D.J.D., S.N.A.). P.M. is supported by the FNRS. G.V. was supported by the FNRS and the Fonds de recherche sur la nature et les technologies (Québec). We thank A. Darsaud, L. James, K. Jones, B. Middleton, C. Phillips, C. Schmidt, V. Sterpenich, and A. Viola for their help.

Correspondence should be addressed to Pierre Maquet, Centre de Recherches du Cyclotron, Université de Liège, B30, Sart Tilman, B-4000 Liège, Belgium. E-mail: pmaquet@ulg.ac.be.

G. Vandewalle's present address: Department of Psychology, Université de Montréal, Montréal QC H2V 2S9, Canada.

DOI:10.1523/JNEUROSCI.0229-09.2009

Copyright $\odot 2009$ Society for Neuroscience $\quad$ 0270-6474/09/297948-09\$15.00/0 differences herein, have not yet been elucidated (Aston-Jones, 2005; Chee and Chuah, 2008).

Previous functional brain imaging studies on the effects of sleep deprivation on cognition yielded a variety of sometimes divergent results (Chee and Chuah, 2008). After sleep deprivation, the most consistent decreases in responses to working memory tasks are observed in posterior (parietal, occipital, temporal) cortices (Chee and Choo, 2004; Habeck et al., 2004), in addition to frontal areas (Habeck et al., 2004; Choo et al., 2005; Mu et al., 2005). In contrast, albeit not always detected (Choo et al., 2005), response increases, which are deemed compensating for the detrimental effects of sleep deprivation, are typically reported in thalamic nuclei (Chee and Choo, 2004; but see Thomas et al., 2000; Habeck et al., 2004), anterior cingulate cortex (Habeck et al., 2004; Choo et al., 2005) and prefrontal areas (Chee and Choo, 2004; Habeck et al., 2004; Mu et al., 2005). Moreover, these regionally specific response patterns appear to differentiate vulnerable participants from subjects resilient to sleep deprivation. Thus, the decrease in parietal activation after sleep deprivation has been proposed as a physiological marker of vulnerability to sleep deprivation ( $\operatorname{Lim}$ et al., 2007). In contrast, sleep deprivation-induced increases in ventral prefrontal cortex are typically reported in the less-vulnerable individuals (Chuah et al., 2006).

This post hoc interpretation of these studies suggests that the 


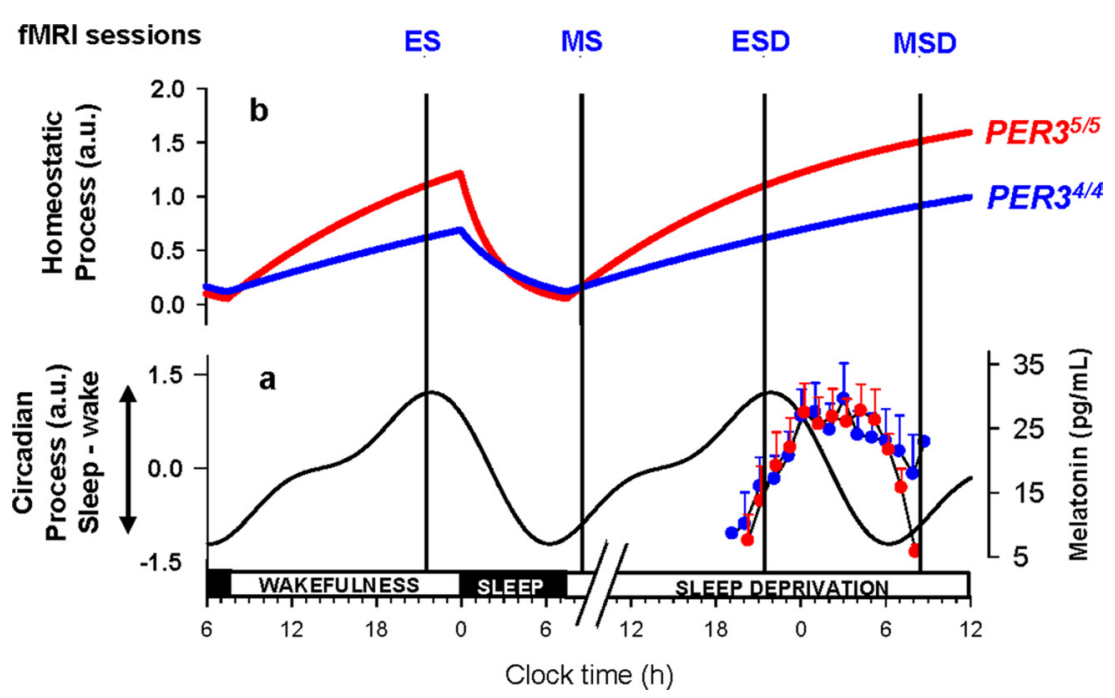

Figure 1. Schematic representation of the protocol and the homeostatic and circadian processes in $P E R 3^{4 / 4}$ and $P E R 3^{5 / 5}$. All times are in clock time (h). a, Circadian arousal signal (Sleep-Wake) [arbitrary units (a.u.)], which promotes wakefulness during the day and sleep at night (based on data published in Dijk et al., 1997) oscillates independently from sleep-wake behavior and similarly in PER3 $3^{4 / 4}$ and $P E R 3^{5 / 5}$. The nadir of the circadian arousal rhythm is located in the early morning and its crest at the end of the habitual waking day. The time course of melatonin during the sleep deprivation condition (means + SE; SEs are not plotted for $n \leq 2$ ) is plotted to the right. $\boldsymbol{b}$, Homeostatic sleep pressure (a.u.) increases during wakefulness, declines during sleep, and increases further during sleep deprivation. Based on waking and sleep EEG data (Viola et al., 2007), homeostatic sleep pressure increases and declines more rapidly in PER3 $3^{5 / 5}$. Vertical lines, Positions of the different $\mathrm{FMRI}$ sessions.

brain response patterns of vulnerable and less-vulnerable individuals dissociate in a predictable way when exposed to sleep loss. A stringent test of this prediction requires a prospective study of brain responses to a working memory task in subjects who are a priori classified as vulnerable or less vulnerable.

We contrasted the dynamics of functional magnetic resonance imaging (fMRI)-assessed brain responses to a working memory task during a normal sleep-wake cycle and after sleep loss, while assessing circadian phase, in a study population stratified according to homozygosity for a variable-number (4 or 5) tandem-repeat primate-specific (Jenkins et al., 2005) polymorphism in the coding region of the clock gene PERIOD3 (PER3) (Archer et al., 2003). This polymorphism confers vulnerability to sleep loss through its effects on sleep homeostasis (Viola et al., 2007). Thus, subjects homozygous for the long repeat $\left(P E R 3^{5 / 5}\right)$ are characterized by a more rapid increase in low frequency EEG oscillations during wakefulness and more slow wave activity during sleep (Viola et al., 2007), and are more vulnerable to deterioration of executive function (Groeger et al., 2008).

\section{Materials and Methods}

More details are presented in the online supplemental material, available at www.jneurosci.org.

Participants. Participants gave their written informed consent and the study was approved by the Ethics Committee of the Faculty of Medicine of the University of Liège. DNA samples were collected and analyzed in 254 right-handed individuals, aged between 18 and 30 years old (supplemental Methods). Among these individuals, 105 and 29 were homozygous for the PER $3^{4}$ and PER $3^{5}$ alleles, respectively. We excluded homozygous individuals with a body mass index $>27$, those who had worked on night shifts during the last year or traveled through more than one time zone during the last 2 months, as well as those who were on medication or consumed psychoactive drugs or excessive caffeine and alcohol (i.e., $>4$ caffeine units/d; $>14$ alcohol units/week -1 unit is equivalent to a halfpint $(220 \mathrm{ml})$ of beer or $1(25 \mathrm{ml})$ measure of spirits or 1 glass $(125 \mathrm{ml})$ of wine). The absence of medical, traumatic, psychiatric or sleep disorders was established in a semistructured interview. Fifteen $P E R 3^{4 / 4}$ and 13 PER $3^{5 / 5}$ individuals were enrolled in the study. They were matched for age and gender, and did not differ with respect to other potential confounders (e.g., mood, anxiety level, IQ, education, see supplemental Table S1, available at www.jneurosci.org as supplemental material). None of these subjects participated in our previous PER3 sleep deprivation study (Viola et al., 2007). One PER $3^{5 / 5}$ individual fell asleep during the fMRI session that followed sleep deprivation (he missed more than half of the trials performance $<20 \%$ ) and was discarded. Therefore, $15 P E R 3^{4 / 4}$ and $12 P E R 3^{5 / 5}$ were included in the analyses (demographics, $\mathrm{PMRI}$ and behavior).

Protocol and theoretical background. The study was conducted between the 15th of November 2006 and the 14th of April 2007 at the Cyclotron Research Centre of the University of Liège. Individuals from both genotypes were recruited to the study throughout the 5 month period. At least $8 \mathrm{~d}$ before the first segment of the experiment, subjects were habituated to sleeping in the laboratory while instrumented for polysomnographic recording. At this time, additional characteristics of the participants were collected, but these were not used as selection criteria (supplemental Table S1, available at www. jneurosci.org as supplemental material).

Each subject participated in two experimental segments (Sleep and Sleep deprivation) separated by at least 1 week (Fig. 1). The order of the segments was counter-balanced across subjects. During the $7 \mathrm{~d}$ preceding each laboratory segment, volunteers were instructed to follow a regular sleep schedule and this was verified using wrist actigraphy (Actiwatch, Cambridge Neuroscience, UK) and sleep diaries (supplemental Table S2, available at www.jneurosci.org as supplemental material). The two segments were identical except for the presence or absence of sleep between the evening and morning fMRI recordings. In one segment, subjects slept in darkness for $7.5 \mathrm{~h}$ (EEG was recorded during the sleep episodes), whereas in the other they stayed awake in dim light throughout the night (a member of the research staff ensured they were awake at all times). The timings of fMRI acquisitions were scheduled with respect to the habitual sleep-schedule, to minimize the confounding effects of variation in sleep-wake timing and circadian phase (see supplemental Table S3, available at www.jneurosci.org as supplemental material, for actigraphy-assessed sleep-wake times). For each laboratory segment, subjects arrived at the laboratory $7.5 \mathrm{~h}$ before and left $6 \mathrm{~h}$ after their scheduled habitual sleep midpoint (Fig. 1). Functional MRI acquisitions were scheduled in the evening, $2 \mathrm{~h}$ before habitual bedtime, i.e., close to the crest of the circadian arousal signal, and in the morning, $1.5 \mathrm{~h}$ after wake time, close to the nadir of the circadian arousal signal (Fig. 1a). This resulted in $4 \mathrm{fMRI}$ sessions: a morning session after sleep (MS; after $\sim 1.5$ $\mathrm{h}$ of wakefulness, at $\sim 08: 30 \mathrm{~h}$ on average), a morning session after sleep deprivation (MSD; after $\sim 25 \mathrm{~h}$ of wakefulness, at $\sim 08: 30 \mathrm{~h}$ on average), an evening session before sleep (ES; after $\sim 14 \mathrm{~h}$ of wakefulness, at $\sim 21: 30 \mathrm{~h}$ on average), and an evening session before sleep deprivation (ESD; after $\sim 14 \mathrm{~h}$ of wakefulness, at $\sim 21: 30 \mathrm{~h}$ on average). Thus, the morning and evening sessions differed with respect to both time awake and circadian phase (see supplemental Results and supplemental Table S3, available at www.jneurosci.org as supplemental material, for precise time awake before each session and clock time of fMRI sessions in each genotype-no significant difference between genotypes were detected, $p>0.1)$. In contrast, the two morning sessions were scheduled at the same circadian phase and differed only with respect to time awake before the session.

The fMRI sessions can also be compared with respect to the previously established difference between the genotypes in the wake-dependent increase and sleep-dependent decline of homeostatic sleep pressure (Viola 
et al., 2007) (Fig. 1b). Based on this established difference, we predicted that, despite near identical wake durations, the change in brain responses over a normal sleep-wake cycle, i.e., the difference between MS and ES, may differ between the genotypes. We further predicted that these genotype-dependent differences would be enhanced after sleep deprivation, i.e., when comparing the difference between MS and MSD.

Description of activities and measurements. Throughout each experimental segment, subjects were maintained in dim light at all times $(<5$ lux), except for the fMRI sessions, which were conducted in nearcomplete darkness ( $<0.01$ lux $)$, and for the sleep episodes, during which they were maintained in complete darkness (0 lux). During sleep deprivation, movements were only allowed at hourly intervals (toilet and stretching), hourly standardized light snacks were provided, and quiet activities were authorized (quiet games, video [ $<5$ lux], and reading). Saliva samples for the determination of the melatonin rhythm were collected once $20 \mathrm{~min}$ before the evening fMRI session and hourly afterward, until the morning fMRI session (11 samples in total) (see supplemental Methods for assays, available at www.jneurosci.org as supplemental material). Activity was strictly controlled for $60 \mathrm{~min}$ before each fMRI session, during which only social interactions were allowed (no reading, snacks, or movements). Subjective alertness scores, as assessed by the Karolinska Sleepiness Scale (KSS) (Akerstedt and Gillberg, 1990), were collected every $30 \mathrm{~min}$ upon arrival and until the end of the protocol the next day, when the participants were awake (i.e., not during the sleep episode of the sleep segment).

In the MR scanner, subjects performed an auditory 3-back task (Cohen et al., 1997), which requires them to state whether or not each auditorily presented consonant was identical to the consonant presented 3 stimuli earlier, using an MR-compatible keypad. They were trained on the task at least a week before the first experimental segment. Seven 3-back task blocks were presented in each session. Sessions lasted 9.5-10 $\mathrm{min}$. The task was kept relatively short $(10 \mathrm{~min})$ to prevent differences between genotypes in the sleep deprivation-induced alterations in performance, which occur if this task is embedded in a longer duration test battery (Groeger et al., 2008).

$f M R I$ data acquisition. Functional MRI time series were acquired using a 3T MR scanner (Allegra, Siemens, Erlangen, Germany). Blood Oxygen Level Dependent (BOLD) signal was recorded using multislice T2* weighted fMRI images, which were obtained with a gradient echo-planar sequence $(\mathrm{EPI})$ using axial slice orientation (32 slices; voxel size: $3.4 \times$ $3.4 \times 3 \mathrm{~mm}^{3}$ with $30 \%$ of gap; matrix size $64 \times 64 \times 32$; repetition time $=2130 \mathrm{~ms}$; echo time $=40 \mathrm{~ms}$; flip angle $=90^{\circ}$. Structural brain images were acquired during the training session and consisted of a T1weighted 3D MDEFT (Deichmann et al., 2004) (repetition time $=7.92$ $\mathrm{ms}$, echo time $=2.4 \mathrm{~ms}$, time of inversion $=910 \mathrm{~ms}$, flip angle $=15^{\circ}$, field of view $230 \times 173 \mathrm{~cm}^{2}$, matrix size $=256 \times 224 \times 173$, voxel size $=$ $1 \times 1 \times 1 \mathrm{~mm}^{3}$ ).

$f M R I$ data analysis. Functional volumes were analyzed using Statistical Parametric Mapping 5 (SPM5; http://www.fil.ion.ucl.ac.uk/spm). They were corrected for head motion, spatially normalized (standard SPM5 parameters) and smoothed. The analysis of fMRI data was conducted in two serial steps, accounting, respectively, for fixed and random effects. For each subject, changes in brain regional responses were estimated using a general linear model, in which the blocks of the 3-back task were modeled using boxcar functions, convolved with a canonical hemodynamic response function. Subject errors (false positives, false negatives and omissions, separately) were modeled using stick functions and convolved with a canonical hemodynamic response function. The regressors derived from errors and realignment of the functional volumes were considered as covariates of no interest. High-pass filtering was implemented in the matrix design using a cutoff period of $128 \mathrm{~s}$ to remove low frequency drifts from the time series. Serial correlations in the fMRI signal were estimated using an autoregressive (order 1) plus white noise model and a restricted maximum likelihood algorithm.

The effects of interest were then tested by linear contrasts in each subject, generating statistical parametric maps. These contrasts of interest included: main effects of the 3-back task during a normal sleep-wake cycle [MS and ES] and after sleep deprivation [MSD]; differences between the evening session and the morning session after sleep in the brain activity related to the 3-back task [MS vs ES]; differences between the morning session after sleep and the morning session after sleep deprivation in the brain activity related to the 3-back task [MS vs MSD]; differences between the evening session and the morning session after sleep deprivation in the brain activity related the 3-back task [MSD vs ESD]. The summary statistic images resulting from these different contrasts were then entered in a second-level analysis accounting for intersubject variance in the effects of interest (random effects model). We first wanted to identify the brain areas involved in the 3-back task during a normal sleep-wake cycle (MS and ES) and after sleep deprivation (MSD) that were common to both genotypes. We therefore computed one-sample $t$ tests for brain responses to the 3-back blocks on one genotype and masked it (inclusive mask thresholded at $p_{\text {uncorrected }}=0.001$ ) by the same one-sample $t$ tests in the other genotype. We then computed twosample $t$ tests for the various contrasts of interest to assess whether these differences were statistically significant across groups.

The resulting set of voxel values for each contrast constituted maps of the $t$ statistics thresholded at $p_{\text {uncorrected }}=0.001$. Statistical inferences were performed after correction for multiple comparisons at a threshold of $p=0.05$. Corrections for multiple comparisons (Family Wise Error method) were based on the Gaussian random field theory and computed on the entire brain volume or on small spherical volumes (10 mm radius) around a priori locations of activation. Activations were expected in structures involved in the n-back tasks, working memory, arousal regulation, or reported in previous investigation of the effects of SD in fMRI or PET (See supplemental Methods for the literature used).

Trait-like sleep propensity was assessed in all subjects at screening using the Epworth Sleepiness Scale (Johns, 1991). Each scale score was assigned to its corresponding subject in a multiple regression analysis at the random effects level on the contrast (summary statistics) images representing the difference between genotypes in the session (morning vs evening) by segment (sleep deprivation vs sleep) interaction [(MSD > $\left.\mathrm{ESD})>(\mathrm{MS}>\mathrm{ES}) *\left(P E R 3^{4 / 4}>P E R 3^{5 / 5}\right)\right]$.

\section{Results}

\section{Sleep-wake timing and melatonin circadian rhythm}

Sleep-wake timing and sleep duration (mean $\pm \mathrm{SD} ; P E R 3^{4 / 4}$, $465 \pm 24 \mathrm{~min} ;$ PER3 $^{5 / 5}, 454 \pm 22 \mathrm{~min}$; supplemental Table S2, available at www.jneurosci.org as supplemental material) before each segment, the duration of wakefulness before each fMRI session (supplemental Table S3, available at www.jneurosci.org as supplemental material), as well as the phase and amplitude of the melatonin rhythm during the night of sleep deprivation-a reliable marker of circadian phase (Klerman et al., 2002) — did not differ between the genotypes $(p>0.1)$ (Fig. 1a; supplemental Results, available at www.jneurosci.org as supplemental material). The average onset of nocturnal melatonin secretion, i.e., the time at which melatonin concentration first exceeds $15 \mathrm{pg} / \mathrm{ml}$, occurred at 20:54 in $P E R 3^{4 / 4}$ and 21:24 in PER3 ${ }^{5 / 5}$.

\section{Changes detected during a normal sleep-wake cycle \\ Behavior}

Subjective sleepiness, as assessed by the KSS (Akerstedt and Gillberg, 1990), and performance on the task did not differ $(p>$ 0.42 ) between the genotypes in the morning (MS) and evening (ES) (Table 1; supplemental Results, supplemental Fig. S1, available at www.jneurosci.org as supplemental material).

\section{Brain responses to the executive task}

Analysis of the brain areas recruited by the task during a normal sleep-wake schedule, i.e., during MS and ES, confirmed that both genotypes recruited areas in frontal, temporal and parietal cortices, areas which are typically involved in working memory (Cohen et al., 1997; Cabeza and Nyberg, 2000; Collette et al., 2005, 2006) (supplemental Fig. S2, supplemental Table S4, available at www.jneurosci.org as supplemental material). 
Table 1. Behavioral results (mean \pm SD)

\begin{tabular}{|c|c|c|c|c|c|c|}
\hline & \multicolumn{2}{|c|}{$\begin{array}{l}\text { Subjective sleepiness (Karolinska } \\
\text { Sleepiness Scale) }\end{array}$} & \multicolumn{2}{|c|}{ Accuracy on the task (\% correct responses) } & \multicolumn{2}{|c|}{ Reaction times (ms) } \\
\hline & $P E R 3^{4 / 4}$ & $P E R 3^{5 / 5}$ & PER3 $3^{4 / 4}$ & $P E R 3^{5 / 5}$ & PER3 $3^{4 / 4}$ & $P E R 3^{5 / 5}$ \\
\hline MS & $3.8 \pm 1.1$ & $3.7 \pm 1.1$ & $89.7 \pm 6.4$ & $88.6 \pm 5.7$ & $1003 \pm 158$ & $1108 \pm 255$ \\
\hline ES & $4.1 \pm 1.4$ & $4.3 \pm 1.3$ & $87.9 \pm 6.2$ & $86.7 \pm 5.2$ & $1060 \pm 156$ & $1162 \pm 242$ \\
\hline ESD & $3.7 \pm 1.2$ & $3.8 \pm 1.2$ & $83.7 \pm 9.4$ & $86.4 \pm 8.8$ & $1123 \pm 204$ & $1133 \pm 250$ \\
\hline MSD & $7.6 \pm 0.8$ & $7.4 \pm 1.6$ & $84.1 \pm 8$ & $82.4 \pm 11.9$ & $1060 \pm 176$ & $1132 \pm 258$ \\
\hline
\end{tabular}
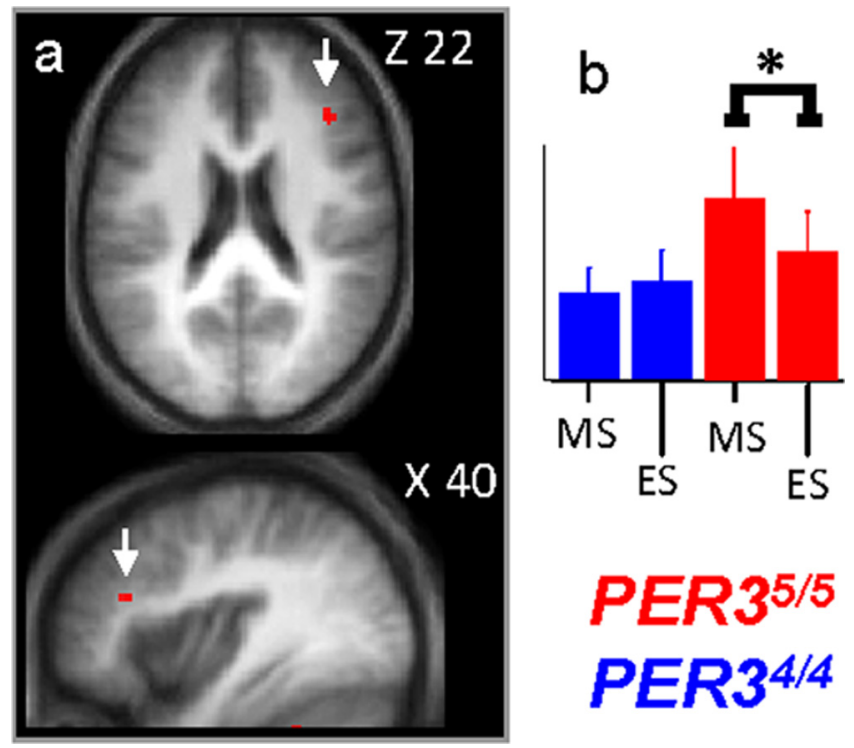

Figure 2. Significant differences in brain response between the sessions recorded after $14 \mathrm{~h}$ and $1.5 \mathrm{~h}$ of wakefulness. $\boldsymbol{a}, P E R 3^{5 / 5}$ significantly reduced response in the right posterior inferior frontal gyrus during ES compared with MS. Statistical results are overlaid to the population mean structural image ( $p_{\text {uncorrected }}<0.001$ ). No significant changes were observed in PER3 $3^{4 / 4}$. $\boldsymbol{b}$, Mean activity estimates (a.u. \pm SEM; in all figures * indicates the significant differences at $p<0.05$ at the voxel level after correction for multiple comparisons).

The dynamics of brain responses in the course of a normal day were then assessed in both genotypes by comparing the evening session to the morning session after sleep. In $P E R 3^{4 / 4}$ participants, no significant changes in brain responses were observed between ES and MS. In contrast, in PER $3^{5 / 5}$, response in the posterior portion of the right inferior frontal gyrus (4032 22; $Z=$ $3.57 ; p_{\text {corrected }}=0.013$ ), was significantly lower in ES compared with MS (Fig. 2). Note that a significant interaction between session $(\mathrm{ES}>\mathrm{MS})$ and genotype $\left(\mathrm{PER}^{4 / 4}>P E R 3^{5 / 5}\right)$ was detected in the exact same brain frontal location.

\section{Changes induced by sleep deprivation \\ Behavior}

Subjective sleepiness was significantly higher in the morning after sleep loss than in the other sessions $(F$-value $>186.28$, $\mathrm{df}=1,25$; $\left.p<10^{-6}\right)$. Both genotypes were, nevertheless, able to maintain performance during the short working memory task, even though greater time-dependent (or within-session) deterioration of performance was detected $(F$-value $=3.39 ; \mathrm{df}=6,150$; $p$-value $=0.004)$. No statistically significant differences $(p>$ 0.18 ) between the genotypes were observed for either performance, or subjective sleepiness (Table 1; supplemental Results, supplemental Fig. S1, available at www.jneurosci.org as supplemental material).
Comparison of brain responses to the executive task in the morning after sleep deprivation to the morning after sleep Similar to the responses observed after a night of sleep, both genotypes recruited areas typically involved in working memory in the morning session after sleep loss (Cohen et al., 1997; Cabeza and Nyberg, 2000; Collette et al., 2005, 2006) (supplemental Fig. S3, supplemental Table S5, available at www.jneurosci.org as supplemental material).

The changes in brain responses resulting from sleep deprivation were then assessed in both genotypes by comparing the morning session after sleep (MS; $1.5 \mathrm{~h}$ of wakefulness) to the morning session after sleep deprivation (MSD; $25 \mathrm{~h}$ of wakefulness). The sleep-deprivation-induced changes in brain responses revealed qualitatively different outcomes in the two genotypes. In the less-vulnerable genotype $\left(P E R 3^{4 / 4}\right)$, significant reductions in activations were not detected in any brain region. In fact, not only was activity in the brain areas typically involved in working memory maintained after sleep deprivation, but activations in supplemental brain areas were observed. Thus, in $P E R 3^{4 / 4}$, sleep deprivation led to activations in the anterior part of the right inferior frontal gyrus. Additional increases in responses were detected in the right middle temporal gyrus, bilateral parahippocampus, superior colliculus, bilateral thalamus areas, and in the left cerebellum (Fig. 3; Table 2). In contrast, in the vulnerable genotype $\left(P E R 3^{5 / 5}\right)$, sleep deprivation led to a reduced activation of the right posterior inferior frontal gyrus, which was already detected in the MS versus ES comparison. In this genotype, sleep deprivation also led to extended reduced activations in the left middle frontal gyrus, bilaterally in the superior temporal sulcus, superior parietal cortex, anterior intraparietal sulcus, and in the middle occipital gyrus (Fig. 3; Table 2). No significant increases in activations in any brain region were found after sleep loss in $P E R 3^{5 / 5}$. It is worth emphasizing that significant interactions between session (MSD > MS) and genotype $\left(P E R 3^{4 / 4}>P E R 3^{5 / 5}\right)$ were detected in most of the brain areas affected by sleep deprivation in each genotype separately in the comparison between MSD and MS (see 1 symbols in Table 2).

Comparison of brain responses to the executive task in the morning after sleep deprivation to the evening before sleep deprivation

Because the comparison between morning after sleep and the morning after sleep loss revealed many more genotypeassociated differences than the comparison between the evening session and the morning session after sleep, the former differences most likely evolved during the night of sleep deprivation. The comparison between the morning after sleep deprivation (MSD) to the evening (ESD) before sleep loss indeed showed that the genotype-dependent response to the effects of sleep deprivation was primarily related to differences in the sensitivity to the overnight extension of wakefulness (Fig. 4; Table 3). In PER $3^{4 / 4}$, comparison of ESD and MSD revealed only increases in activation, and these were located in the left thalamus, left parahippocampus and left cerebellum. In contrast, in $P E R 3^{5 / 5}$, the comparison of ESD and MSD revealed reduced activations in a large 
number of cortical and subcortical areas, including frontal, parietal, occipital cortices. Again, note that significant interactions between session (MSD > ESD) and genotype $\left(\right.$ PER $\left.3^{4 / 4}>P E R 3^{5 / 5}\right)$ were detected in most of the brain areas affected by sleep deprivation in each genotype separately in the comparison between MSD and ESD (see 9 symbols in Table 3).

Changes in thalamic activity elicited by sleep deprivation is related to a trait-like sleepiness measure

We next investigated whether the differential dynamics of the brain responses could be related to an individual's self-estimated likelihood of falling asleep while living in their normal environment. A trait-like sleep propensity estimate was assessed in all participants at screening (i.e., at least a week before the first fMRI segment and before the $7 \mathrm{~d}$ of regular sleep) using the Epworth Sleepiness Scale (ESS), which is a well validated scale of the propensity to fall asleep during a variety of nonstimulating waking activities (Johns, 1991). ESS scores in $P E R 3^{5 / 5}$ individuals $(6.96 \pm 1.07)$ were significantly higher than in $P E R 3^{4 / 4}$ individuals $(3.70 \pm 0.64)(p=0.008)$. This constituted the only significant difference between the two genotypes at screening. (supplemental Table S1, available at www.jneurosci.org as supplemental material).

In an analysis in which the two genotype groups were pooled, this sleep propensity measure correlated significantly and negatively with the difference in the overnight change in activity in the thalamus on the nights with and without sleep (Fig. 3, yellow circle; $-22,-28,4, \mathrm{Z}=$ $\left.3.36, p_{\text {corrected }}=0.019\right)$. Subjects with higher self-reported sleep propensity underwent greater overnight thalamic deactivation after sleep loss, compared with after a night of sleep. Although we conducted this analysis independent of genotype, the genotypes were segregated in the correlation between sleep propensity and fMRI data because of their different average ESS scores. We therefore infer that $P E R 3^{5 / 5}$ individuals, with the highest selfreported sleep propensity, underwent the greater thalamic deactivation after sleep loss, compared with $P E R 3^{4 / 4}$.

\section{Discussion}

The data establish that maintenance of executive functions in the course of a normal waking day and after sleep loss is associated with changes in regional brain response patterns to an executive task. As predicted, despite near-identical wake durations, these changes differ markedly between vulnerable and less-vulnerable genotypes, which have been shown to differ with respect to markers of sleep homeostasis (Viola et al., 2007) and executive function

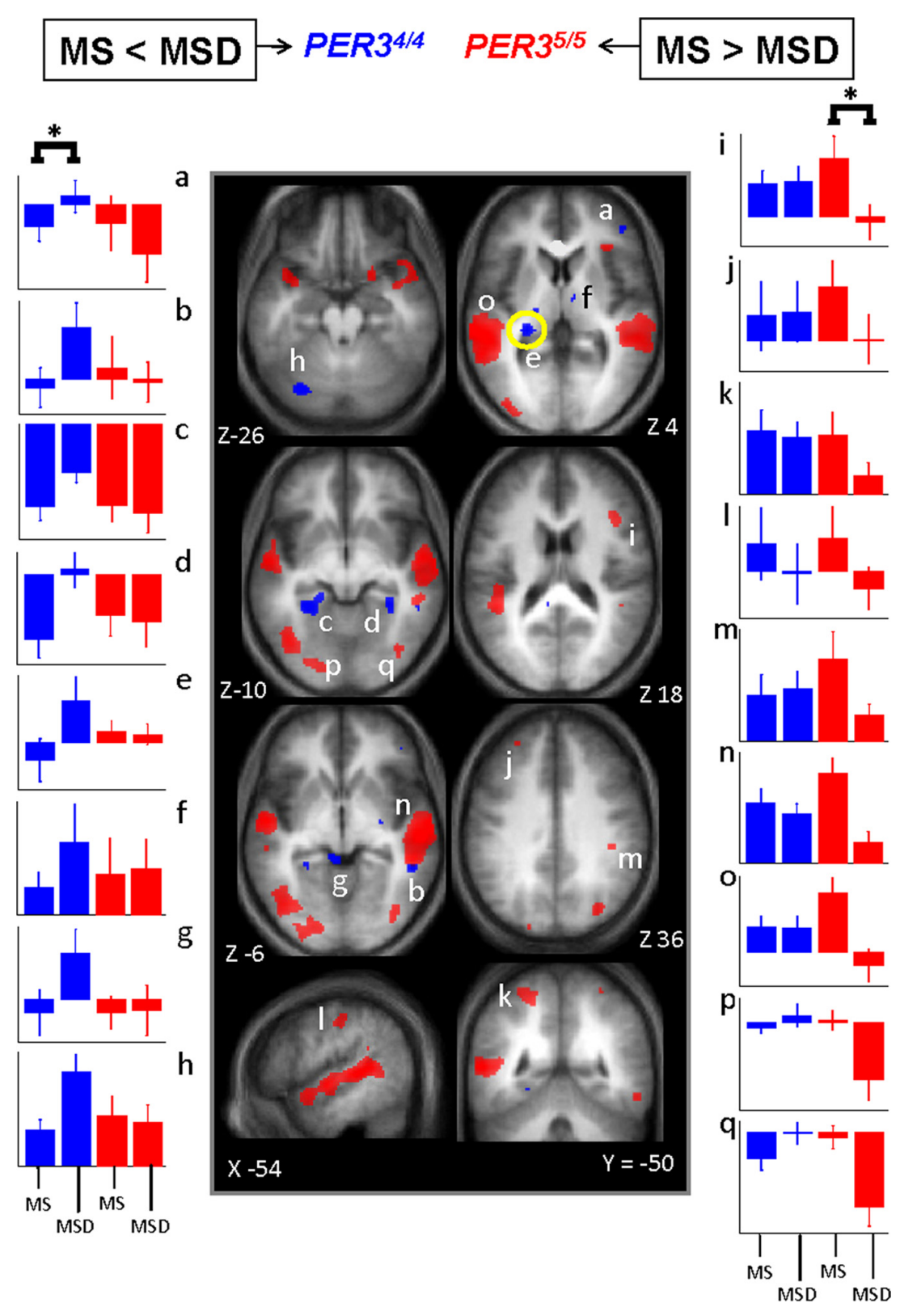

Figure 3. Significant differences in brain response between the sessions recorded after $25 \mathrm{~h}$ and $1.5 \mathrm{~h}$ of wakefulness. Middle panels, In PER3 $3^{4 / 4}$ (blue), only significant increased activations were found in MSD compared with MS. In PER3 $3^{5 / 5}$ (red), only significant reduced activations were found in MSD compared with MS (see Table 2 for the names corresponding to the letters in the structural image). The difference in overnight change in activity in the area circled in yellow (thalamus; Z 4 brain slice) on the nights with and without sleep was significantly and negatively correlated with the self-assessed likelihood to fall asleep in nonstimulating situations during a normal waking day. Lateral panels, Mean activity estimates (a.u. \pm SEM). Note that in the left panels $(\boldsymbol{a}-\boldsymbol{h})$, for all areas indicated by an asterisk $\left({ }^{*}\right)$, there were significant increases in activation, i.e., only in PER $3^{4 / 4}$, whereas in the right panels $(\boldsymbol{i}-\boldsymbol{q})$ for all areas indicated by an asterisk $\left({ }^{*}\right)$, there were decreases in activation, i.e., only in PER $3^{5 / 5}$. Note that in the middle frontal gyrus $(i)$, difference is also significant between MS and ES as reported in Figure 2.

(Groeger et al., 2008). The differential brain responses are present during a normal waking day, become more pronounced in the morning after sleep loss, occur before performance differences emerge and, in accordance with our previous study (Viola et al., 2007; Archer et al., 2008), are observed in the absence of differences in melatonin circadian rhythm.

Neural correlates of vulnerability to sleep loss are detected in PER $3^{5 / 5}$ participants only

In participants a priori classified as vulnerable, sleep deprivation induced marked reductions of activation in posterior prefrontal 


\begin{tabular}{|c|c|c|c|c|c|}
\hline Brain areas & Side & $X, Y, Z$ & Zscore & Brodmann's area & $p_{\text {corrected }}$ value \\
\hline \multicolumn{6}{|l|}{ Increase in brain activity in $P E R 3^{4 / 4}$ (MSD $\left.>M S\right)$} \\
\hline Inferior frontal gyrus (a) & $\mathrm{R}$ & $40,42,0$ & 3.94 & 46 & $0.003^{1, \#}$ \\
\hline Middle temporal gyrus $(b)$ & $\mathrm{R}$ & $54,-44,-6$ & 3.26 & 21 & $0.023^{1, \#}$ \\
\hline \multirow[t]{2}{*}{ Parahippocampus $(c, d)$} & $\mathrm{L}$ & $-30,-34,-14$ & 3.91 & & $0.003^{1, \#}$ \\
\hline & $\mathrm{R}$ & $30,-38,-8$ & 3.18 & & 0.028 \\
\hline \multirow[t]{4}{*}{ Thalamus $(e, f)$} & $\mathrm{L}$ & $-26,-30,6$ & 3.81 & & $0.005^{\#}$ \\
\hline & $\mathrm{L}$ & $-14,-24,10$ & 3.39 & & 0.016 \\
\hline & $\mathrm{L}$ & $-20,-18,4$ & 3.25 & & 0.022 \\
\hline & R & $8,-8,4$ & 3.27 & & 0.024 \\
\hline Superior colliculus $(g)$ & $\mathrm{L}$ & $-4,-40,-4$ & 4.00 & & 0.002 \\
\hline Cerebellum $(h)$ & $\mathrm{L}$ & $-32,-76,-26$ & 3.80 & & $0.005^{1, \#}$ \\
\hline \multicolumn{6}{|l|}{ Decrease in brain activity in PER3 $3^{4 / 4}$ (MSD $<M S$ ) } \\
\hline \multicolumn{6}{|l|}{ No significant voxels $\left(p_{\text {uncorrected }}<0.001\right)$} \\
\hline \multicolumn{6}{|l|}{ Increase in brain activity in PER $3^{5 / 5}$ (MSD > MS) } \\
\hline \multicolumn{6}{|l|}{ No significant voxels $\left(p_{\text {uncorrected }}<0.001\right)$} \\
\hline \multicolumn{6}{|l|}{ Decrease in brain activity in PER $3^{5 / 5}$ (MSD $<M S$ ) } \\
\hline \multirow[t]{2}{*}{ Middle frontal gyrus $(i, j)$} & $\mathrm{R}$ & $44,24,22$ & 3.58 & $44 / 45$ & $0.009^{9, \#}$ \\
\hline & $\mathrm{L}$ & $-30,46,34$ & 3.15 & 9 & 0.030 \\
\hline \multirow[t]{3}{*}{ Superior parietal cortex $(k)$} & $\mathrm{L}$ & $-24,-52,60$ & 3.55 & 7 & $0.010^{9, \#}$ \\
\hline & $\mathrm{L}$ & $-24,-62,52$ & 3.91 & 7 & 0.003 \\
\hline & $\mathrm{R}$ & $30,-48,66$ & 3.14 & 7 & $0.031^{1, \#}$ \\
\hline \multirow[t]{2}{*}{ Postcentral gyrus/anterior intraparietal sulcus $(I, m)$} & $\mathrm{L}$ & $-54,-22,40$ & 3.10 & 40 & 0.035 \\
\hline & $\mathrm{R}$ & $42,-32,36$ & 3.31 & 40 & $0.020^{\#}$ \\
\hline \multirow[t]{5}{*}{ Superior temporal sulcus $(n, 0)$} & $\mathrm{L}$ & $-64,-34,4$ & 4.64 & 22 & $0.05^{\pi}$ \\
\hline & $\mathrm{L}$ & $-56,-46,8$ & 4.29 & $21 / 22$ & $0.011^{\#}$ \\
\hline & $\mathrm{L}$ & $-56,6,-20$ & 3.52 & 21 & 0.001 \\
\hline & $\mathrm{R}$ & $60,-22,-4$ & 4.64 & 22 & 0.05 \\
\hline & $\mathrm{R}$ & $54,-38,-2$ & 4.08 & 21 & $0.002^{1, \#}$ \\
\hline \multirow[t]{5}{*}{ Middle occipital gyrus $(p, q)$} & $\mathrm{L}$ & $-44,-72,-8$ & 3.87 & 37 & $0.013^{9, \#}$ \\
\hline & L & $-22,-88,-6$ & 3.46 & 18 & $0.004^{9, \#}$ \\
\hline & L & $-44,-86,4$ & 3.37 & 37 & $0.017^{\Uparrow}$ \\
\hline & $\mathrm{L}$ & $-34,-90,-6$ & 3.23 & 18 & $0.025^{1, \#}$ \\
\hline & $\mathrm{R}$ & $40,-76,-8$ & 3.47 & 37 & $0.013^{\mathbb{1}}$ \\
\hline
\end{tabular}

Letters in parentheses $(a-q)$ : refer to Figure 3. L, Left; $R$, right.

"Significant interaction between session and genotype: [(MSD $>M S) *\left(\right.$ PER $3^{4 / 4}>$ PER $\left.\left.3^{5 / 5}\right)\right]$.

"Significant interaction between session, segment, and genotype: $\left[(M S D>E S D)>(M S>E S) *\left(P E R 3^{4 / 4}>P E R 3^{5 / 5}\right)\right]$ (see supplemental Table S6, available at www.jneurosci.org as supplemental material, for complete results of this interaction).

cortices after sleep loss, which was already detected in the evening after a normal waking day but became particularly pronounced during circadian phase misalignment, i.e., in the morning after sleep loss. In these individuals, parietal and temporal cortices undergo marked reductions of activation after sleep loss. Because we used the auditory modality, this supports the assumption that deficits in short-term memory after sleep deprivation result, in part, from impaired attention and sensory processing (Chee and Chuah, 2007; Chee et al., 2008). Likewise, occipital areas, which were not activated by the task during a normal sleep-wake cycle, were deactivated after sleep loss. Given the absence of visual input in our design, this is likely to reflect the diminution of top-down influences of associative (parietal and frontal) cortices onto occipital regions. Surprisingly, the substantial decreases in brain responses observed in $P E R 3^{5 / 5}$ individuals were not detected in $P E R 3^{4 / 4}$. In the latter genotype, responses in these brain regions after sleep loss were essentially unchanged. These conspicuous differences in response patterns between the genotypes are most likely related to the differential effects of sleep loss in interaction with circadian misalignment, and not to simple differences in the circadian modulation of brain responses. This is because in the morning after sleep, i.e., when sleep pressure is low in both genotypes, this genotype-dependent reduced activation pattern was not observed. In fact, in $P E R 3^{5 / 5}$, responses in most prefrontal and posterior regions are largest in the morning shortly after sleep and then decline in the course of a normal waking day, and in the morning after sleep loss these areas are barely activated (Figs. 3i,j, $m-o, 4 d-j$ ).

\section{Neural correlates of resilience to sleep loss are detected in PER $^{4 / 4}$ participants only}

Sleep loss triggered increases in brain responses, but, again unexpectedly, only in the $P E R 3^{4 / 4}$ genotype. These individuals, a priori classified as less vulnerable on the basis of their genotype, seem to maintain cognition in response to increasing homeostatic sleep pressure through two separate mechanisms: 1) the recruitment of supplemental areas (including an anterior prefrontal area), and 2) the maintenance of posterior and anterior cortices activity.

A ventral anterior prefrontal area, which is not engaged by the task under conditions of rested wakefulness, is recruited after sleep deprivation, suggesting that novel cognitive strategies are developed to maintain performance in the adverse cognitive conditions resulting from sleep loss. A recent model of hierarchical organization of prefrontal cortex suggests that premotor areas map simple sensory-motor responses, intermediate dorsolateral prefrontal regions control responses related to the current context, and higher-order anterior ventral prefrontal regions modu- 
late behavior in the context of past events (Koechlin et al., 2003). A more 'episodic' behavioral strategy may well represent an efficient alternative strategy to maintain performance to the task. Additional brain areas recruited after sleep loss (cerebellum, superior colliculus) are known to interact with frontal areas in the context of tasks engaging working memory and attention (Prabhakaran et al., 2000; Shipp, 2004). We speculate that their recruitment during the morning session after sleep loss reflects a direct influence of frontal areas.

The lack of thalamic activation during sleep loss in $P E R 3^{5 / 5}$ underscores the importance of the functional interaction between thalamus and cortex, which is further supported by the correlation between thalamic activation and the self-assessed likelihood to fall asleep in nonstimulating situations during a normal waking day. Thus, genotype-dependent changes after sleep loss, as well as trait-like sleep propensity, can be related to differences in thalamic activity. It may, therefore, be in the thalamus that homeostatic and circadian changes affect the regulation of cognition and alertness (Aston-Jones, 2005). Previous fMRI studies, manipulating arousal using pharmacological agents [caffeine (Portas et al., 1998), noradrenergic A2 agonists (Coull et al., 2004), sleep deprivation (Portas et al., 1998)], or a circadian factor [light exposure (Vandewalle et al., 2006)], associated arousal with thalamic activity while performing cognitive tasks.

\section{Changes in regional brain responses precede behavioral modifications}

It may be surprising that, despite substantial differences in brain activations, performance of both genotypes was not yet different. However, in both groups, performance was still supported by the recruitment of a common distributed set of areas. In fact, the data suggest that in the course of a normal sleep-wake cycle and during sleep loss, changes in circadian phase and homeostatic sleep pressure lead to changes in brain responses, which to some extent differ between genotypes, before differences in performance emerge. Nevertheless, we speculate that these different response patterns will lead to differences in behavior when vigilance is further challenged by extending the duration of wakefulness or of the tasks (Viola et al., 2007). In this respect, decreased alertness probably participates in performance deterioration to the task, which is unlikely to result only from specific effects of sleep loss on executive functions.

In our design, baseline activity was assessed during interleaved resting periods. Changes in baseline rest activity could lead to apparent changes in brain activation in response to the task. An active reference condition (0- or 1-back task), would have allowed better control over baseline activity across session but would have lengthened fMRI sessions, thereby increasing the likelihood of behavioral differences between genotypes, reported when using longer test-batteries after sleep deprivation (Viola et al., 2007). Even if baseline changes were partly responsible for the effects we report, it remains that they were clearly different between genotypes.

\section{Sleep-wake regulation, circadian rhythmicity, and regional} brain function

All our observations are consistent with an inverted ' $U$ ' view, in which the increased homeostatic sleep pressure associated with sustained wakefulness initially leads to activation of higher-order 'episodic' frontal areas and increased recruitment of arousalrelated thalamic regions. Further extension of wakefulness and associated increase in homeostatic sleep pressure, in conjunction with circadian misalignment, then leads to reduced activation not only in executive frontal areas, but also in cortical areas distributed across parietal, temporal and occipital cortices. Because homeostatic sleep pressure, as measured by EEG slow wave activity during NREM sleep and EEG theta activity during wakefulness, increases more rapidly in $P E R 3^{5 / 5}$ (Viola et al., 2007), the loss of executive control occurs after fewer hours of wakefulness in this genotype, and may already arise during a normal waking day, but becomes particularly pronounced as soon as the circadian arousal signal dissipates. This earlier loss of executive control may indeed provide brain bases for individual differences in 
Table 3. Significant differences between sessions recorded after $25 \mathrm{~h}$ (MSD) and $14 \mathrm{~h}$ (ESD) of wakefulness in brain activity related to the 3-back task in PER3 $3^{4 / 4}$ and PER3 $3^{5 / 5}$ genotypes

\begin{tabular}{|c|c|c|c|c|c|}
\hline Brain areas & Side & $X, Y, Z$ & Brodmann's area & $Z$ & $p_{\text {corrected }}$ value \\
\hline \multicolumn{6}{|l|}{ Increase in brain activity in PER3 $3^{4 / 4}$ (MSD > ESD) } \\
\hline Thalamus $(a)$ & L & $-6,-12,8$ & & 3.10 & $0.039^{\pi}$ \\
\hline Parahippocampus (b) & $\mathrm{L}$ & $-24,-42,-10$ & & 3.13 & $0.036^{\natural}$ \\
\hline Cerebellum (c) & $\mathrm{L}$ & $-30,-76,-26$ & & 3.26 & $0.026^{\pi}$ \\
\hline \multicolumn{6}{|l|}{ Decrease in brain activity in $P E R 3^{4 / 4}$ (MSD $<$ ESD) } \\
\hline \multicolumn{6}{|l|}{ Increase in brain activity in PER $3^{5 / 5}$ (MSD > ESD) } \\
\hline \multicolumn{6}{|l|}{ Decrease in brain activity in $P E R 3^{5 / 5}$ (MSD $\left.<E S D\right)$} \\
\hline Inferior frontal gyrus (d) & $\mathrm{L}$ & $-54,32,12$ & 46 & 4.75 & $0.038^{9}$ \\
\hline \multirow[t]{3}{*}{ Inferior frontal gyrus/middle frontal gyrus (e) } & $\mathrm{R}$ & $46,32,4$ & 46 & 4.67 & $0.05^{\pi}$ \\
\hline & R & $44,32,14$ & 46 & 3.93 & 0.003 \\
\hline & $\mathrm{R}$ & $40,18,22$ & $44 / 45$ & 3.92 & 0.004 \\
\hline Superior frontal sulcus & $\mathrm{L}$ & $-22,54,0$ & 10 & 3.60 & 0.010 \\
\hline \multirow[t]{4}{*}{ Superior parietal cortex $(f, g)$} & $\mathrm{L}$ & $-24,-54,62$ & 7 & 3.87 & 0.004 \\
\hline & $\mathrm{L}$ & $-26,-64,56$ & 7 & 3.80 & 0.005 \\
\hline & R & $24,-52,60$ & 7 & 3.48 & 0.018 \\
\hline & R & $14,-54,66$ & 7 & 3.33 & $0.022^{9}$ \\
\hline \multirow[t]{2}{*}{ Postcentral gyrus/anterior intraparietal sulcus $(h, i)$} & $\mathrm{R}$ & $42,-32,40$ & 40 & 4.08 & 0.002 \\
\hline & $\mathrm{L}$ & $-38,-24,42$ & 40 & 3.39 & 0.018 \\
\hline \multirow[t]{3}{*}{ Superior temporal sulcus $(j, k)$} & $\mathrm{L}$ & $-52,12,-26$ & 21 & 4.85 & $0.026^{\pi}$ \\
\hline & L & $-52,-48,8$ & $21 / 22$ & 4.65 & $<0.001^{\pi}$ \\
\hline & $\mathrm{R}$ & $52,-36,-8$ & 21 & 4.02 & $0.003^{q}$ \\
\hline \multirow[t]{5}{*}{ Middle occipital gyrus $(I, m)$} & R & $30,-86,-4$ & 19 & 4.42 & $0.002^{9}$ \\
\hline & R & $32,-84,10$ & 19 & 3.63 & $0.009^{\pi}$ \\
\hline & $\mathrm{L}$ & $-38,-72,-2$ & $19 / 37$ & 4.36 & $0.001^{\natural}$ \\
\hline & $\mathrm{L}$ & $-24,-86,-4$ & 18 & 4.12 & $0.001^{\pi}$ \\
\hline & L & $-22,-76,18$ & 19 & 3.47 & $0.015^{\pi}$ \\
\hline \multirow[t]{2}{*}{ Fusiform gyrus } & R & $42,-58,-18$ & $19 / 37$ & 4.07 & $0.002^{\pi}$ \\
\hline & R & $28,-66,-12$ & 19 & 3.67 & 0.008 \\
\hline \multirow[t]{2}{*}{ Cingulate cortex (n) } & L & $-10,-18,36$ & $24 / 31$ & 3.87 & $0.004^{9}$ \\
\hline & R & $16,46,10$ & 32 & 3.43 & 0.016 \\
\hline Insula (o) & $\mathrm{L}$ & $-38-1012$ & & 3.73 & 0.007 \\
\hline Putamen $(p)$ & L & $-22,2,2$ & & 3.50 & $0.013^{\pi}$ \\
\hline Globus pallidus & $\mathrm{R}$ & $20,0,0$ & & 3.35 & $0.020^{9}$ \\
\hline
\end{tabular}

Letters in parentheses $(a-v)$ : Reference to Figure 4. L, Left; $R$, right.

"Significant interaction between session and genotype: [(MSD > ESD $) *\left(P E R 3^{4 / 4}>\right.$ PER3 $\left.\left.{ }^{5 / 5}\right)\right]$.

vulnerability to sleep loss. This interpretation leads to several testable predictions. For example, we expect in $P E R 3^{4 / 4}$ to find extended reduced activation if sleep loss were prolonged further than in the current protocol.

Previous studies have emphasized interindividual variability in brain responses after sleep loss. "Compensatory" responses to cognitive tasks have been reported (Chee and Choo, 2004; Drummond et al., 2004; Habeck et al., 2004), whereas maintenance of performance was associated with activations in prefrontal and parietal cortices, and deterioration of performance was associated with loss of activation in these areas (Mu et al., 2005; Chee et al., 2006; Lim et al., 2007). Some of the variability previously observed may be related to genotypic variations in PER3, among other genes.

The PER3 gene is expressed in the suprachiasmatic nucleiloci of the circadian pacemaker-but also in other hypothalamic, subcortical and cortical areas (Takumi et al., 1998), which could all contribute to the effects we observed.

The current observation that differences between genotypes are primarily located between the evening session before and the morning session after sleep loss, and the general observation that the BOLD signal changed very little in the course of a normal waking day, indicates that the BOLD signal does not simply reflect variables that increase monotonically with the duration of wakefulness, e.g., EEG low frequency oscillations (Dijk et al., 1987) or synaptic potentiation (Vyazovskiy et al., 2008). Instead, the time course of the BOLD signal appears more similar to the time courses of variables that are determined by an interaction of time awake and circadian phase, such as performance, which remains near stable during a normal waking day and then declines rapidly during the biological night, and much more so in PER3 $^{5 / 5}$ (Dijk et al., 1992; Viola et al., 2007; Groeger et al., 2008). Identifying the cellular mechanisms responsible for these BOLD differences may provide tools to uncover the nature of the interaction of homeostatic and circadian signals, as well as individual differences in the vulnerability to sleep loss.

\section{References}

Akerstedt T, Gillberg M (1990) Subjective and objective sleepiness in the active individual. Int J Neurosci 52:29-37.

Archer SN, Robilliard DL, Skene DJ, Smits M, Williams A, Arendt J, von Schantz M (2003) A length polymorphism in the circadian clock gene Per3 is linked to delayed sleep phase syndrome and extreme diurnal preference. Sleep 26:413-415.

Archer SN, Viola AU, Kyriakopoulou V, von Schantz M, Dijk DJ (2008) Inter-individual differences in habitual sleep timing and entrained phase of endogenous circadian rhythms of BMAL1, PER2 and PER3 mRNA in human leukocytes. Sleep 31:608-617.

Aston-Jones G (2005) Brain structures and receptors involved in alertness. Sleep Med 6 (Suppl 1):S3-S7. 
Cabeza R, Nyberg L (2000) Imaging cognition II: An empirical review of 275 PET and fMRI studies. J Cogn Neurosci 12:1-47.

Cajochen C, Wyatt JK, Czeisler CA, Dijk DJ (2002) Separation of circadian and wake duration-dependent modulation of EEG activation during wakefulness. Neuroscience 114:1047-1060.

Chee MW, Choo WC (2004) Functional imaging of working memory after $24 \mathrm{hr}$ of total sleep deprivation. J Neurosci 24:4560-4567.

Chee MW, Chuah LY (2008) Functional neuroimaging insights into how sleep and sleep deprivation affect memory and cognition. Curr Opin Neurol 24:417-423.

Chee MW, Chuah YM (2007) Functional neuroimaging and behavioral correlates of capacity decline in visual short-term memory after sleep deprivation. Proc Natl Acad Sci U S A 104:9487-9492.

Chee MW, Chuah LY, Venkatraman V, Chan WY, Philip P, Dinges DF (2006) Functional imaging of working memory following normal sleep and after 24 and $35 \mathrm{~h}$ of sleep deprivation: correlations of fronto-parietal activation with performance. Neuroimage 31:419-428.

Chee MW, Tan JC, Zheng H, Parimal S, Weissman DH, Zagorodnov V, Dinges DF (2008) Lapsing during sleep deprivation is associated with distributed changes in brain activation. J Neurosci 28:5519-5528.

Choo WC, Lee WW, Venkatraman V, Sheu FS, Chee MW (2005) Dissociation of cortical regions modulated by both working memory load and sleep deprivation and by sleep deprivation alone. Neuroimage 25:579-587.

Chuah YM, Venkatraman V, Dinges DF, Chee MW (2006) The neural basis of interindividual variability in inhibitory efficiency after sleep deprivation. J Neurosci 26:7156-7162.

Cohen JD, Perlstein WM, Braver TS, Nystrom LE, Noll DC, Jonides J, Smith EE (1997) Temporal dynamics of brain activation during a working memory task. Nature 386:604-608.

Collette F, Van der Linden M, Laureys S, Delfiore G, Degueldre C, Luxen A, Salmon E (2005) Exploring the unity and diversity of the neural substrates of executive functioning. Hum Brain Mapp 25:409-423.

Collette F, Hogge M, Salmon E, Van der Linden M (2006) Exploration of the neural substrates of executive functioning by functional neuroimaging. Neuroscience 139:209-221.

Coull JT, Jones ME, Egan TD, Frith CD, Maze M (2004) Attentional effects of noradrenaline vary with arousal level: selective activation of thalamic pulvinar in humans. Neuroimage 22:315-322.

Czeisler CA, Dijk DJ (2001) Human circadian physiology and sleep-wake regulation. In: Handbook of behavioral neurobiology: circadian clocks. (Takahashi JS, Turek FW, Moore RY, eds), pp 531-561. New York: Kluwer Academic/Plenum.

Deichmann R, Schwarzbauer C, Turner R (2004) Optimisation of the 3D MDEFT sequence for anatomical brain imaging: technical implications at 1.5 and $3 \mathrm{~T}$. Neuroimage 21:757-767.

Dijk DJ, von Schantz M (2005) Timing and consolidation of human sleep, wakefulness, and performance by a symphony of oscillators. J Biol Rhythms 20:279-290.

Dijk DJ, Beersma DG, Daan S (1987) EEG power density during nap sleep: reflection of an hourglass measuring the duration of prior wakefulness. J Biol Rhythms 2:207-219.

Dijk DJ, Duffy JF, Czeisler CA (1992) Circadian and sleep/wake dependent aspects of subjective alertness and cognitive performance. J Sleep Res 1:112-117.

Dijk DJ, Shanahan TL, Duffy JF, Ronda JM, Czeisler CA (1997) Variation of electroencephalographic activity during non-rapid eye movement and rapid eye movement sleep with phase of circadian melatonin rhythm in humans. J Physiol 505:851-858.

Drummond SP, Brown GG, Salamat JS, Gillin JC (2004) Increasing task difficulty facilitates the cerebral compensatory response to total sleep deprivation. Sleep 27:445-451.

Groeger JA, Viola AU, Lo JC, von Schantz M, Archer SN, Dijk DJ (2008) Early morning executive functioning during sleep deprivation is compromised by a PERIOD3 polymorphism. Sleep 31:1159-1167.

Habeck C, Rakitin BC, Moeller J, Scarmeas N, Zarahn E, Brown T, Stern Y (2004) An event-related fMRI study of the neurobehavioral impact of sleep deprivation on performance of a delayed-match-to-sample task. Brain Res Cogn Brain Res 18:306-321.

Jenkins A, Archer SN, von Schantz M (2005) Expansion during primate radiation of a variable number tandem repeat in the coding region of the circadian clock gene period3. J Biol Rhythms 20:470-472.

Johns MW (1991) A new method for measuring daytime sleepiness: the Epworth sleepiness scale. Sleep 14:540-545.

Klerman EB, Gershengorn HB, Duffy JF, Kronauer RE (2002) Comparisons of the variability of three markers of the human circadian pacemaker. J Biol Rhythms 17:181-193.

Koechlin E, Ody C, Kouneiher F (2003) The architecture of cognitive control in the human prefrontal cortex. Science 302:1181-1185.

Lim J, Choo WC, Chee MW (2007) Reproducibility of changes in behaviour and fMRI activation associated with sleep deprivation in a working memory task. Sleep 30:61-70.

Mu Q, Mishory A, Johnson KA, Nahas Z, Kozel FA, Yamanaka K, Bohning DE, George MS (2005) Decreased brain activation during a working memory task at rested baseline is associated with vulnerability to sleep deprivation. Sleep 28:433-446.

Portas CM, Rees G, Howseman AM, Josephs O, Turner R, Frith CD (1998) A specific role for the thalamus in mediating the interaction of attention and arousal in humans. J Neurosci 18:8979-8989.

Prabhakaran V, Narayanan K, Zhao Z, Gabrieli JD (2000) Integration of diverse information in working memory within the frontal lobe. Nat Neurosci 3:85-90.

Shipp S (2004) The brain circuitry of attention. Trends Cogn Sci 8:223-230.

Takumi T, Taguchi K, Miyake S, Sakakida Y, Takashima N, Matsubara C, Maebayashi Y, Okumura K, Takekida S, Yamamoto S, Yagita K, Yan L, Young MW, Okamura H (1998) A light-independent oscillatory gene mPer3 in mouse SCN and OVLT. EMBO J 17:4753-4759.

Thomas M, Sing H, Belenky G, Holcomb H, Mayberg H, Dannals R, Wagner H, Thorne D, Popp K, Rowland L, Welsh A, Balwinski S, Redmond D (2000) Neural basis of alertness and cognitive performance impairments during sleepiness. I. Effects of $24 \mathrm{~h}$ of sleep deprivation on waking human regional brain activity. J Sleep Res 9:335-352.

Vandewalle G, Balteau E, Phillips C, Degueldre C, Moreau V, Sterpenich V, Albouy G, Darsaud A, Desseilles M, Dang-Vu TT, Peigneux P, Luxen A, Dijk DJ, Maquet P (2006) Daytime light exposure dynamically enhances brain responses. Curr Biol 16:1616-1621.

Van Dongen HP, Baynard MD, Maislin G, Dinges DF (2004) Systematic interindividual differences in neurobehavioral impairment from sleep loss: evidence of trait-like differential vulnerability. Sleep 27:423-433.

Viola AU, Archer SN, James LM, Groeger JA, Lo JC, Skene DJ, von Schantz M, Dijk DJ (2007) PER3 polymorphism predicts sleep structure and waking performance. Curr Biol 17:613-618.

Vyazovskiy VV, Cirelli C, Pfister-Genskow M, Faraguna U, Tononi G (2008) Molecular and electrophysiological evidence for net synaptic potentiation in wake and depression in sleep. Nat Neurosci 11:200-208. 